\title{
ON THE RELATION BETWEEN POLARIMETRIC SYNTHETIC APERTURE RADAR (SAR) FEATURES AND SEA ICE MELT POND FRACTION
}

\author{
Ane S. Fors ${ }^{1}$, Anthony P. Doulgeris ${ }^{1}$, Angelika H.H. Renner ${ }^{2,3}$, Camilla Brekke ${ }^{1}$ and Sebastian Gerland ${ }^{2}$ \\ ${ }^{1}$ University of Troms $\varnothing$, Department of Physics and Technology, 9037 Troms $\varnothing$, Norway \\ ${ }^{2}$ Norwegian Polar Institute, FRAM Center, 9296 Troms $\varnothing$, Norway \\ ${ }^{3}$ Institute of Marine Research, 9294 Troms $\varnothing$, Norway
}

\begin{abstract}
In this study we examine the relationship between sea ice melt pond fraction and two polarimetric C-band synthetic aperture radar (SAR) features. Three high-resolution SAR scenes were recorded in the Fram Strait in late summer 2011, covering iceberg-fast first-year and old sea ice. In addition, melt pond fraction was retrieved from photographs recorded during a helicopter flight at the study area. Co-polarisation ratio and relative kurtosis were extracted from the SAR scenes and compared to the retrieved melt pond fraction. A correlation between relative kurtosis and melt pond fraction was found in all scenes. Co-polarisation ratio was correlated to melt pond fraction in one of the scenes. Refreezing of the melt ponds could explain the lack of correlation in the two other scenes. Our results demonstrate a potential of high resolution C-band SAR for retrieval of melt pond fraction, a valuable parameter in climate model parametrization and climate studies.
\end{abstract}

Index Terms - SAR, polarimetry, sea ice, melt pond

\section{INTRODUCTION}

Knowledge of sea ice melt pond fraction is important in climate model parameterization and in understanding the mass and energy budgets of the ocean-sea ice-atmosphere system[1]. Information about the number and extent of melt ponds and variation in melt pond fraction during the melt season is sparse. Recently, algorithms for retrieval of melt pond fraction have been developed for multi-spectral optical satellites [2], however the use of optical data is often limited by persistent cloud cover in the Arctic in summer. Synthetic aperture radar (SAR) offers all-weather sea ice monitoring, but only a limited number of studies have investigated the possibilities of extraction of melt pond fraction from SAR $[3,4$, and references therein]. To our knowledge, the most promising previous study demonstrates good estimates of melt pond fraction from an empirical model (CV-model) based on the SAR feature co-polarisation ratio [3].

In this study we examine the relationship between melt pond fraction and co-polarisation ratio in three high resolution C-band SAR scenes captured on first and multi year sea ice in the Framstrait during late summer 2011. We evaluate the performance of the CV-model developed in [3] on our dataset. In addition, we look at the relationship between melt pond fraction and relative kurtosis, a statistical SAR feature sensitive to mixed surfaces [5]. Relative kurtosis has showed a promising relationship to melt pond fraction in our dataset [6]. The performance of the two features is compared, and their potential for sea ice melt pond fraction extraction from late summer sea ice is evaluated.

\section{DATA COLLECTION}

The dataset used in this study was collected during a campaign in the Fram Strait in late summer 2011 (Fig. 1). The study area consists of ponded first and multiyear sea ice in different stages of development. The sea ice was held in place by grounded icebergs and did not move significantly during the data collection period.

Table 1. Overview of data collection

\begin{tabular}{llll}
\multicolumn{3}{c}{ Table 1. Overview of data collection } \\
\hline Date & Time (UTC) & Scene ID & $\begin{array}{l}\text { Incidence } \\
\text { angle }\end{array}$ \\
\hline $29 / 08 / 2011$ & $17: 41$ & R1 & $38.2^{\circ}$ \\
$31 / 08 / 2011$ & $18: 23$ & R2 & $48.2^{\circ}$ \\
$03 / 09 / 2011$ & $14: 09$ & Helicopter flight & - \\
$04 / 09 / 2011$ & $18: 07$ & R3 & $44.4^{\circ}$ \\
\hline
\end{tabular}

Three full polarimetric C-band SAR scenes cover the study area (Table 1). The scenes are all ascending fine quadpolarimetric mode scenes acquired from Radarsat-2. In addition, downward looking photographs were taken during a helicopter flight at the study area during the campaign. A semi-automatic method was used to extract melt pond fraction from the photographs [7]. Due to the low temperatures, the melt pond fraction is considered constant during the week of study. The retrieved melt pond fraction is therefore considered valid in all the three satellite scenes investigated. At the time of the campaign, the melt ponds had started to refreeze, and there was likely a mix of open and refrozen melt ponds in the study area. It was not possible to access the study site 


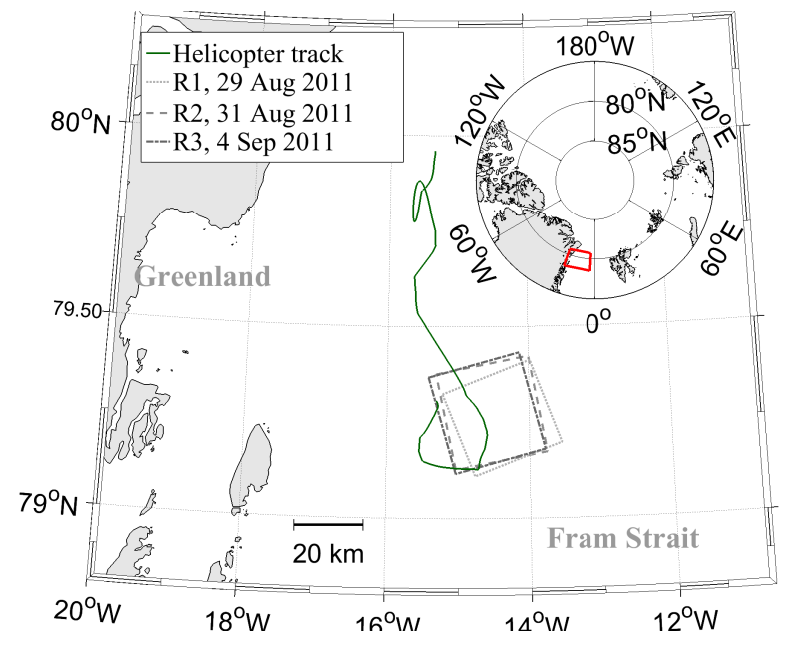

Fig. 1. Map of the western Fram Strait with the location of the satellite scenes (gray frames) and the track of the helicopter flight collecting melt pond fraction (green line). The star symbol represents the position of R/V Lance at the time of the helicopter flight. The red box in the inset map of the northern hemisphere shows the position of the area displayed.

to perform ground-based measurements during the campaign, hence the degree of refreezing is unknown.

Meteorological measurements were recorded on the research vessel R/V Lance, sailing close to the study area (Fig. 2). As a validity check, we extracted $2 \mathrm{~m}$ air temperature from the European Centre for Medium-Range Weather Forcasts (ECMWF) ERA-interim re-analysis, both for the position of the satellite scenes $\left(14.25^{\circ} \mathrm{W} 79.25^{\circ} \mathrm{N}\right)$ and for R/V Lance's position.

\section{POLARIMETRIC SAR FEATURES}

From the satellite scenes, co-polarisation ratio and relative kurtosis were extracted with a $7 \times 7$ pixels non-overlapping stepping window, giving $L=49$ number of looks.

Co-polarisation ratio $\left(R_{V V / H H}\right)$ is the ratio between the $S_{V V}$ and the $S_{H H}$ polarimetric channel, defined as:

$$
R_{V V / H H}=\frac{<S_{V V} S_{V V}^{*}>}{<S_{H H} S_{H H}^{*}>},
$$

where $\langle\cdot\rangle$ denotes an average over the number of looks and the asterix denotes the complex conjugate. It is sensitive to differences in the complex dielectric permittivity of the surface, and can hence separate saline sea ice from fresh water. The predictive CV-model developed in [3] suggest melt pond fraction, $f_{p}$, to be related to co-polarisation ratio by

$$
f_{p}=0.1525 \times R_{V V / H H}+0.1564 .
$$

where $R_{V V / H H}$ is given in $\mathrm{dB}$. The model gave good estimates of melt pond fraction for open melt ponds on first-year ice in the central Canadian Arctic Archipelago [3].
Relative kurtosis $(R K)$ is a statistical measure of nonGaussianity, used to describe the shape of the distribution of the scattering coefficient in SAR scenes [5]. It is defined as

$$
R K=\frac{1}{L} \frac{1}{d(d+1)} \sum_{i=1}^{L}\left[\mathbf{s}_{i}^{* T} \mathbf{C}^{-1} \mathbf{s}_{i}\right]^{2},
$$

where $d$ is the number of polarimetric channels. $\mathbf{s}_{i}$ is the Lexicografic feature vector $\left(\mathbf{s}_{i}=\left[\begin{array}{lll}S_{H H} & \sqrt{2} S_{V H} & S_{V V}\end{array}\right]^{T}\right)$ and $\mathbf{C}$ is the covariance matrix $\left(C=\frac{1}{L} \sum_{i=1}^{L} s_{i} s_{i}^{* T}\right)$. Relative kurtosis is expected to be sensitive to mixtures of surfaces, and could therefore have a potential in melt pond fraction retrieval. A relative kurtosis of one is associated with a homogeneous surface with Gaussian distribution of the complex scattering coefficient. On the other hand, a relative kurtosis different from one is associated with a heterogeneous surface with a non-Gaussian distribution of the complex scattering coeficient, either a distribution with a sharp peak and heavy tails $(R K>1)$ or a broad distribution with light tails $(R K<1)$.

\section{METHODS}

Mean values of melt pond fraction and of the two SAR features were extracted from 50 sections of $400 \mathrm{~m}$ length along the track of the helicopter flight. The values were compared in scatter plots to examine the relationship between the melt pond fraction and the SAR features in the three investigated scenes. Spearman's correlation coefficient $\left(\rho_{s}\right)$ was used to evaluate the correlation. It takes values between 0 and \pm 1 , where 0 corresponds to no correlation, and \pm 1 corresponds to full correlation. A negative sign indicates an inverse relationship. $\rho_{s}$ makes the assumption of a monotonic relationship, and is less sensitive to outliers than, e.g., Pearson's correlation coefficient describing linear relationships.

\section{RESULTS AND DISCUSSION}

Whether the melt ponds were open or refrozen at the time of the acquisition of the satellite scenes is important for our results. The air temperature measurements could give an indication of the state of the ponds. Focusing on the air temperature measured at $\mathrm{R} / \mathrm{V}$ Lance, it was below $-1^{\circ} \mathrm{C}$ the days prior to and at the acquisition time of R1 and R3 (Fig 2). $\mathrm{R} 2$ was acquired after a period with air temperatures ranging from $-1^{\circ} \mathrm{C}$ to $0.5^{\circ} \mathrm{C}$. The ERA-Interim modelled air temperatures were higher than the measured air temperature, but had only small numerical differences between the position of the satellite scenes and R/V Lance. This supports the use of the measured air temperature at R/V Lance also for the position of the satellite scenes. From these observations, the melt ponds are expected to be refrozen at the acquisition time of $\mathrm{R} 1$ and R3. At the acquistion time of R2 there is a possiblilty of open ponds. 


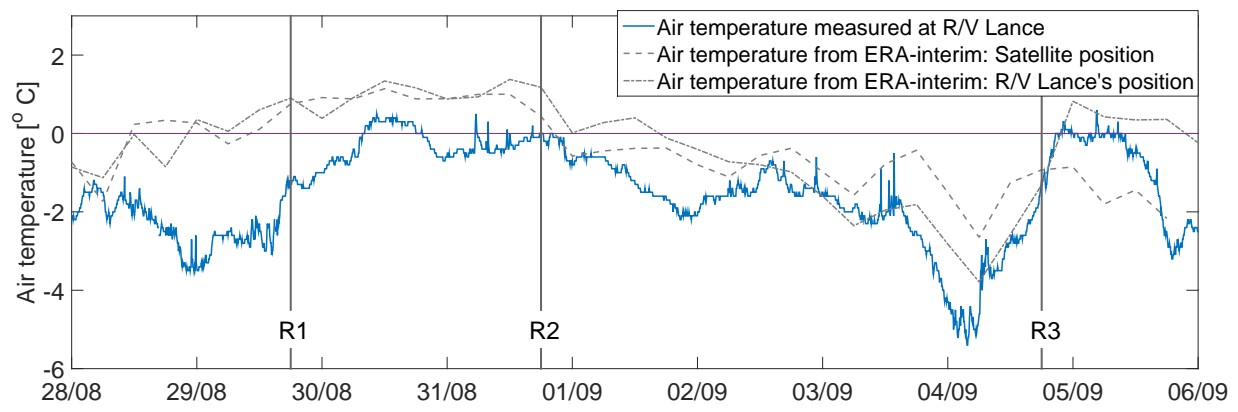

Fig. 2. Air temperature during the campaign. The blue line is measurements recorded at R/V Lance, and the red dots represent time of the acquisition of the Radarsat- 2 scenes. The gray dotted lines are reanalyed air temperature from ECWMF meteorological data, retrieved from ERA interim for the position of the satellite scenes and R/V Lance. The lilac colored line represents zero degree Celcius.

\subsection{Co-polarisation ratio and melt pond fraction}

Co-polarisation ratio displayed a medium good correlation to melt pond fraction in R2 $\left(\rho_{s}=0.59\right)$, increasing with melt pond fraction (Figure 3(b)). No significant correlation was found in R1 and R3 (Fig. 3(a) and (c)). Co-polarisation ratio is expected to be insensitive to melt pond fraction when the melt ponds are refrozen, as refrozen melt ponds have a copolariation ratio similar to that of bare sea ice [3]. The lack of relation between melt pond fraction and co-polarisation ratio in R1 and R3 points towards refrozen melt ponds in these scenes. This is supported by the temperature measurements.

\subsection{Relative kurtosis and melt pond fraction}

Relative kurtosis was correlated with melt pond fraction in all three scenes investigated ( $\rho_{s}$ were $-0.61,-0.62$ and -0.68 , respectively), decreasing with increasing melt pond fraction (Fig. 3 (d)-(f)). The areas with low melt pond fraction have a relative kurtosis above one, while the areas with higher melt pond fraction have values below one. The values are comparable in all three scenes.

\subsection{The CV model}

The CV-model was evaluated with data from R2. The model is represented by the dashed line in Fig. 3(b). The root mean square error of the model is large, with a value 0.2192 , and the slope is too steep compared to the data. The model was developed from smooth first-year ice in spring [3], while the sea ice in our study is rougher and has lower salinity due to seasonal desalination. These differences could possibly explain the offset between the CV-model and our data. Rougher sea ice exceeds the Bragg limit, and weakens the relationship between co-polarisation and the dielectric constant, and less saline ice causes less contrast between the dielectric constant of sea ice and melt ponds. In addition, the ponds might be partly refrozen, lowering their co-polarisation ratio.

\subsection{Relative kurtosis vs. co-polarisation ratio}

The degree of correlation between relative kurtosis and melt pond fraction was similar to or sligthly higher than that found between co-polarisation ratio and melt pond fraction in $\mathrm{R} 2$. Co-polarisation ratio was not related to melt pond fraction in R1 and R3, most probably because of refrozen melt ponds, while relative kurtosis seemed to be insensitive to the state of the ponds.

Despite differences, both SAR features were found to be correlated to melt pond fraction in parts of the investigated dataset. The CV-model failed to give a good estimate of melt pond fraction, but with adjustment to season, site and sea ice type, a model based on co-polarisation ratio has potential for open melt pond fraction extraction. The correlation between relative kurtosis and melt ponds highlights the possibility of using statistical SAR features in melt pond fraction retrieval.

\section{CONCLUSIONS}

Melt pond fraction is an important sea ice parameter in climate model parametrisation, but has so far been poorly mapped. SAR could have a potential in melt pond fraction retrieval. In this study, the SAR features co-polarisation ratio and relative kurtosis were found related to melt pond fraction in late summer. For co-polarisation the relationship was limited to one scene, probably due to refrozen melt ponds in the two other investigated scenes. The results show that SAR features both based on dielectric and statistical properties of the sea ice surface have a potential in melt pond fraction retrieval in late summer. However, more data and further investigations are needed to fully reveal the potential of melt pond fraction extraction from the two features.

\section{ACKNOWLEDGEMENTS}

Thanks to the captain, crew and scientists from the Norwegian Polar Institute onboard R/V Lance in the Fram Strait 


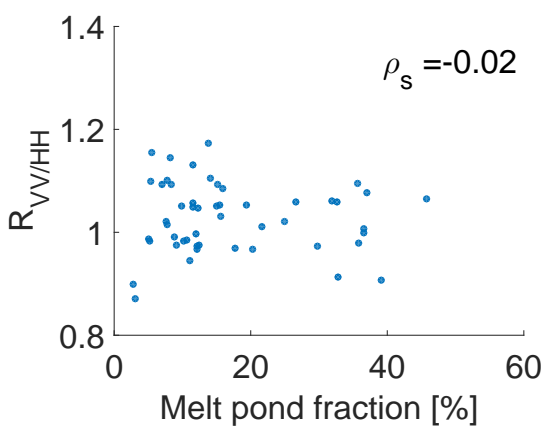

(a) R1

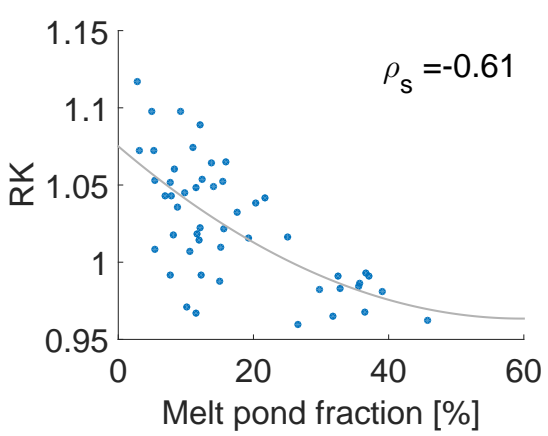

(d) R1

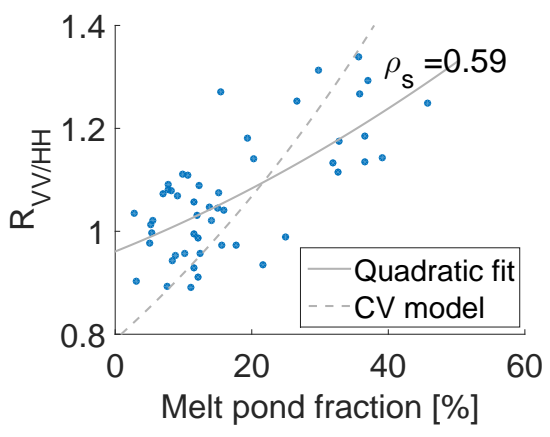

(b) R2

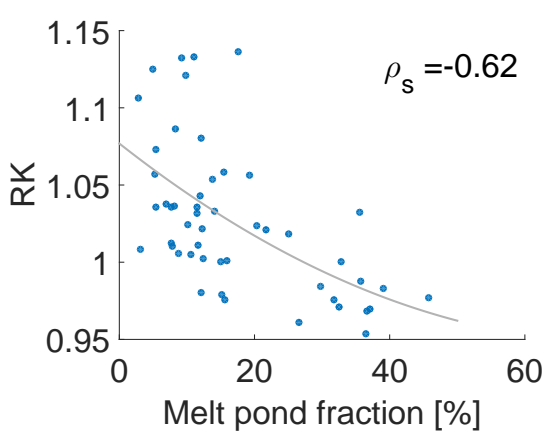

(e) R2

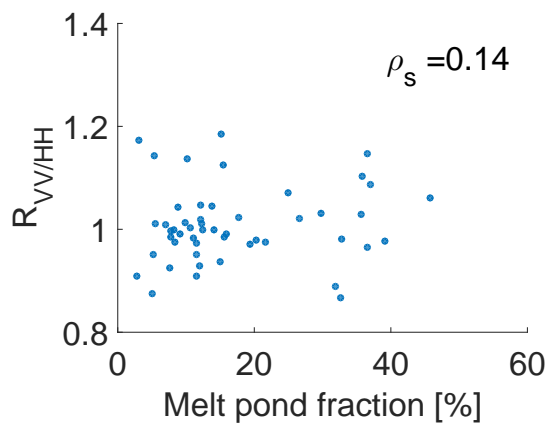

(c) R3

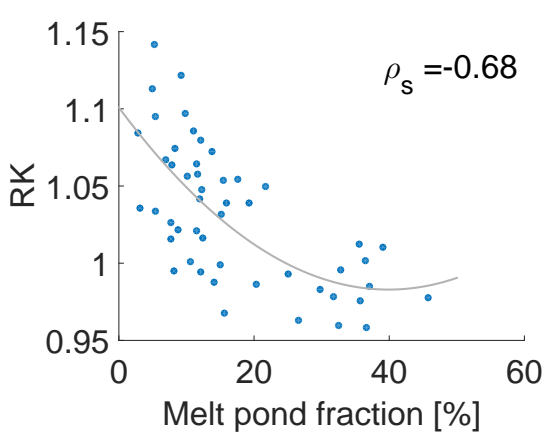

(f) R3

Fig. 3. Scatter plots of melt pond fraction versus co-polarisation ratio $\left(R_{V V / H H}\right)$ (top) and melt pond fraction versus relative kurtosis $(R K)$ (bottom) for the three included SAR scenes (R1, R2 and R3). The values are calculated as the mean values of co-located sections of 400 meters length along the track of the helicopter. Fit lines are quadratic. Spearman's correlation coefficient $\left(\rho_{s}\right)$ measure the strength of the relationship, and clearly (a) and (c) show no correlation.

2011 for data collection. Radarsat-2 data are provided by NSC/KSAT under the Norwegian-Canadian Radarsat agreement 2011. This project was supported financially by the project "Sea Ice in the Arctic Ocean, Technology and Systems of Agreements" ("Polhavet", subproject "CASPER") of the Fram Centre, by the Centre for Ice, Climate and Ecosystems at the Norwegian Polar Institute and by RDA Troms County.

\section{REFERENCES}

[1] C. Polashenski, D. Perovich, and Z. Courville, "The mechanisms of sea ice melt pond formation and evolution," Journal of Geophysical Research, vol. 117, no. C1, pp. C01001, Jan. 2012.

[2] A. Rösel, L. Kaleschke, and G. Birnbaum, "Melt ponds on Arctic sea ice determined from MODIS satellite data using an artificial neural network," The Cryosphere, vol. 6, no. 2, pp. 431-446, Apr. 2012.

[3] R. K. Scharien, K. Hochheim, J. Landy, and D. G. Barber, "First-year sea ice melt pond fraction estimation from dual-polarisation C-band SAR Part 2: Scaling in situ to Radarsat-2," The Cryosphere, vol. 8, no. 6, pp. 2163-2176, Nov. 2014.

[4] M. Makynen, S. Kern, A. Rösel, and L. T. Pedersen, "On the Estimation of Melt Pond Fraction on the Arctic Sea Ice With ENVISAT WSM Images," IEEE Transactions on Geoscience and Remote Sensing, vol. 52, no. 11, pp. 7366-7379, Nov. 2014.

[5] M.-A. Moen, A. P. Doulgeris, S. N. Anfinsen, A. H. H. Renner, N. Hughes, S. Gerland, and T. Eltoft, "Comparison of feature based segmentation of full polarimetric SAR satellite sea ice images with manually drawn ice charts," The Cryosphere, vol. 7, no. 6, pp. 1693-1705, Nov. 2013.

[6] A. Fors, C. Brekke, S. Gerland, A. P. Doulgeris, and T. Eltoft, "Extraction of late summer sea ice properties from polarimetric SAR features in C- and X-band," in Proc. POLinSAR. 2015, 2015.

[7] A. H. H. Renner, M. Dumont, J. Beckers, S. Gerland, and C. Haas, "Improved characterisation of sea ice using simultaneous aerial photography and sea ice thickness measurements," Cold Regions Science and Technology, vol. 92, pp. 37-47, Aug. 2013. 